\title{
Ultrasonic Imaging of Concrete by Synthetic Aperture Focusing Technique Based on Hilbert-Huang Transform of Time Domain Data
}

\author{
Oscar Victor M. Antonio, Jr. ${ }^{1,2, *}$ and Sohichi Hirose ${ }^{2}$ \\ ${ }^{1}$ Institute of Civil Engineering, University of the Philippines, Diliman, Quezon City 1101, Philippines \\ ${ }^{2}$ Department of Mechanical and Environmental Informatics, Graduate School of Information Science and Engineering, \\ Tokyo Institute of Technology, Tokyo 152-8550, Japan
}

For non-destructive evaluation (NDE) of concrete, efficient and accurate imaging techniques are needed for a reliable evaluation of safety and serviceability of structures. Imaging of concrete structures is considered a very challenging task since it is a non-homogeneous material. In this paper, the acoustic (stress wave) technique was applied by using Ultrasonics and Synthetic Aperture Focusing Technique (SAFT).

In ultrasonic NDE, the presence of coarse aggregates requires that the ultrasonic testing be conducted at relatively low frequencies in order to avoid excessive attenuation. The Hilbert-Huang Transform (HHT), based on Ensemble Empirical Mode Decomposition (EEMD), was used as a signal processing tool for interpreting and analyzing the ultrasonic waveforms taken from a concrete specimen with an embedded steel rod. SAFT images for both processed and unprocessed signals were obtained and compared. Results showed that the reflections from the bottom surface as well as the steel rod were successfully imaged when the processed signal were used. Therefore, anomaly or defect detection in concrete structures through ultrasonic imaging was greatly improved by the combination of HHT and SAFT.

[doi: $10.2320 /$ matertrans.I-M2012804]

(Received November 12, 2009; Accepted March 11, 2011; Published February 29, 2012)

Keywords: ultrasonic imaging, signal processing, non-homogeneous material, sifting, backpropagation, Hilbert-Huang transform

\section{Introduction}

For non-destructive evaluation (NDE) of concrete, efficient and accurate imaging techniques are needed for a reliable evaluation of safety and serviceability of structures. Imaging of concrete structures for non-destructive testing is defined in a broad sense to obtain a representation of certain physical properties of concrete material and characteristics of the physical system by indirect or remote sensing methods which will not damage the structure, or permanently impair its serviceability. ${ }^{1)}$ Since concrete is a highly non-homogeneous material, imaging of concrete structures is considered a very challenging task. Most of the time concrete is produced in the field under very limited quality control. Also, grain size distribution is highly variable and the properties of the constituent materials are greatly varied, thus, making it difficult to obtain accurate images. The presence of coarse aggregate, often exceeding $10 \mathrm{~mm}$ in diameter, requires that the ultrasonic testing in concrete be conducted at relatively low frequencies in order to avoid excessive attenuation caused by scattering. ${ }^{1)}$

At present, there are several techniques used in imaging concrete structures. These include X-ray and gamma-ray radiography, computerized radioactive tomography based on X-rays and gamma-rays, infrared thermography, radar (microwave) and acoustic (stress wave) techniques. In this paper, the acoustic (stress wave) technique is applied by using Ultrasonics and Synthetic Aperture Focusing Technique (SAFT). In Ultrasonic Testing (UT), an ultrasonic pulse is generated by exciting a piezoelectric material with a highamplitude, transient electrical pulse from a high-voltage and high current pulser. The short burst of ultrasonic energy is transmitted into the concrete and impinges upon various

*Graduate Student, Tokyo Institute of Technology. Present address: University of the Philippines interfaces (or inclusions) within the concrete. The change in acoustic impedance at the various interfaces causes some portions of the input energy to reflect or echo back to the surface. $^{2)}$ These energy reflections are then received and converted back to electrical pulse as waveform data.

In concrete UT, particularly for indirect transmission tests, the resulting waveforms are almost always characterized by several oscillations (multi-mode arrival times) due to the reflections from various scatterers within the concrete. Reflections from these multiple scatterers make the interpretation of the waveform very difficult. Thus, digital signal processing (DSP) is needed in order to aid in the interpretation of these waveforms. DSP is used to decompose the waveforms into its simple oscillatory components and to eliminate noise or any unnecessary components. Also, signal processing is very important to time-frequency analysis for better understanding and interpretation of the waveforms as well as the resulting images. In this study, a relatively new signal processing method called Hilbert-Huang Transform (HHT) was used. HHT is easier to implement than a prioribased methods like Wavelet Analysis (WA). It should be noted that the results of WA are highly dependent on the choice of mother wavelet and these results are meaningful only when the mother wavelet used is appropriate for the problem $^{3)}$ (a priori).

\subsection{Hilbert-Huang transform}

The Hilbert-Huang Transform is an empirically-based adaptive data-analysis method developed by Huang et al. ${ }^{4)}$ Its development was motivated by the need to describe in detail the nonlinear distorted waves. Being adaptive means that the definition of the basis function has to be datadependent, an a posteriori-defined basis, an approach totally different from the established mathematical paradigm for data analysis. Its algorithm consists of two parts: Mode Decomposition (MD) and Hilbert Spectral Analysis (HSA). HHT 
method is potentially viable for nonlinear and non-stationary data analysis, especially for time-frequency-energy representations. It has been tested and validated exhaustively, but only empirically. ${ }^{5}$ )

The mode decomposition part serves as a filter that sifts through the data and breaks it down into simpler components called Intrinsic Mode Function (IMF). The sifting operation involves getting the mean envelope from the upper and lower envelopes of the original dataset and then subtracting it from the original dataset. An IMF is produced if the number of extrema differs from the number of zero crossings by not more than one and the mean value of the envelopes is zero. The data returned as IMF is stored and subracted from the parent data. The difference is then used as a parent dataset for a new sifting process and the procedure is repeated until the residue becomes a monotonic function, from which no more IMF can be extracted. After obtaining all the IMFs, the HSA procedure is applied to each IMF to obtain the instantaneous frequency and consequently the frequency-energy distribution as a function of time.

\subsection{Synthetic aperture focusing technique}

Synthetic Aperture Focusing Technique (SAFT) is based on a backpropagation technique which produces an image of the object interior by focusing the recorded data. Its algorithm numerically superimposes signals measured at several positions, thus creating a high-resolution image. 2D SAFT is an integration of the time of flight (TOF) in the $x-y-t$ data field for each element (pixel) of the specimen. It results in a two dimensional representation of the backscatter intensity from the interior of the specimen. Signals are focused to every image point and structural noise is suppressed by spatial superposition. ${ }^{6}$ Ultrasonic SAFT reconstruction was adopted from optical holography and synthetic aperture techniques in radar [Flaherty et al., U.S. Patent No. $3,548,642$ (1970)]. It has been successfully used for imaging homogeneous materials such as steel and aluminum. In the case of reinforced concrete, SAFT image is expected to provide information about thickness variations as well as the inclusions such as the reinforcing bars, cracks, voids, delaminations, and deteriorated zones.

\section{Methodology}

\subsection{Data gathering}

In this study, a concrete block specimen with the following dimensions: $L=575 \mathrm{~mm}, W=575 \mathrm{~mm}$, and $H=120 \mathrm{~mm}$, was used to illustrate the application of 2D SAFT for ultrasonic image reconstruction. The concrete specimen has a $38 \mathrm{~mm}$ diameter duct at the middle, with a $32 \mathrm{~mm}$ steel rod grouted inside (Fig. 1). The ultrasonic testing apparatus consisted of the following: a high power tone burst pulser (Ritec RF Gated Amplifier), a broad band receiver (Ritec BR-640), a function generator (Agilent 33250A), a microcomputer based digital oscilloscope (Hewlett Packard Infinium Oscilloscope), and two broad band transducers with central frequency of $100 \mathrm{kHz}$. The function generator was set to produce a sine wave (single cycle) with frequency of $100 \mathrm{kHz}$. The short burst of longitudinal ultrasonic wave is transmitted into the concrete using a Japan Probe PS 936 (a)

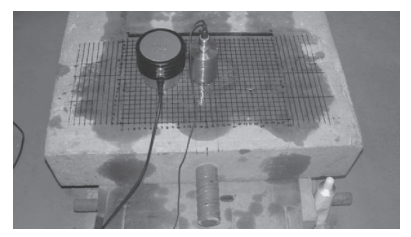

(b)

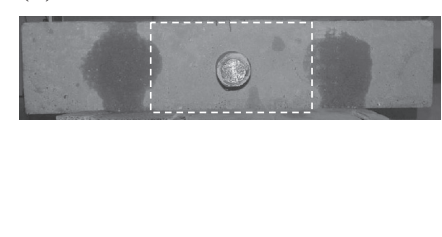

Fig. 1 Concrete block specimen. (a) Pitch-catch method conducted on the top surface of the specimen, (b) Target area for image reconstruction.

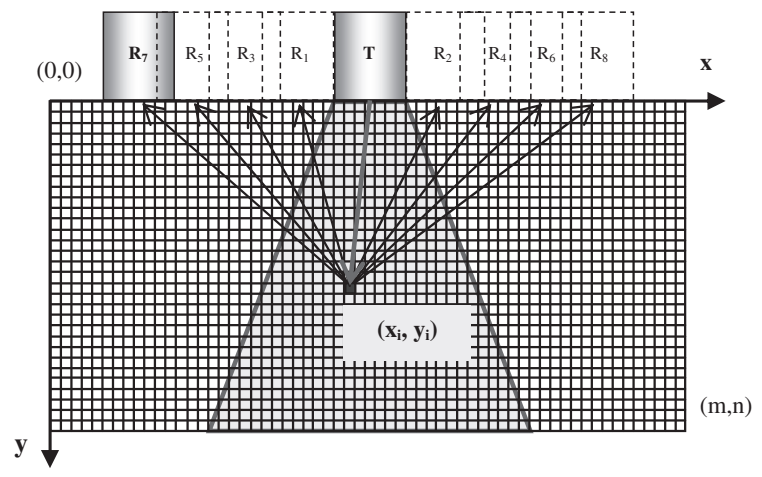

Fig. 2 Schematic diagram for 2D Pitch-Catch SAFT.

transducer (diameter $=55 \mathrm{~mm}$ ) and correspondingly received by a KGK K-85 transducer (diameter $=76 \mathrm{~mm}$ ). A total of 168 waveform data were recorded along a linear aperture on the top surface of the specimen by Pitch-Catch method. The position of the transmitting transducer was changed at increments of $10 \mathrm{~mm}$ and for each transmitter positions, eight readings (four on the left and four on the right) were obtained from the receiving transducer as shown in Fig. 2. The center-to-center distances of the transmitting and receiving transducers were $70,80,90$ and $100 \mathrm{~mm}$. The sampling frequency was set at $5.0 \mathrm{MSa} / \mathrm{s}(5 \mathrm{MHz})$ and obtained 1,002 data points for each waveform. The averaging method (16 waveforms) was implemented for the data acquisition in order to reduce the coherent signal noise. A band pass filter was used in order to suppress the low and high frequency vibrations. The lower and higher cut-off frequencies were at $50 \mathrm{kHz}$ and $3 \mathrm{MHz}$, respectively. Moreover, aside from using a coupling gel, a steel block weighing $3.17 \mathrm{~kg}$ was placed on top of each of the transducers to assure good acoustical coupling.

Before conducting the pitch-catch method, the ultrasonic velocity of the concrete block was obtained by measuring the travel time of the stress wave along a known dimension of the specimen. Using the transmitting transducer (PS 936), the ultrasonic pulse was sent to the receiving transducer (K-85) through the specimen. A cross-correlation procedure was performed between the transmitted and received signals in order to obtain the lag time, which was used to compute for the phase velocity.

\subsection{Signal processing}

All the 168 received waveforms were normalized with respect to each individual input signals. These normalized waveforms were then subjected to Hilbert-Huang Transform in order to obtain the intrinsic mode functions and the 
corresponding frequency as a function of time. Described below is the detailed algorithm in performing the HHT.

\subsubsection{Mode decomposition (MD)}

\section{(a) Empirical Mode Decomposition (EMD)}

Empirical mode decomposition is a sifting process to decompose the waveform data into its intrinsic modes. The decomposition is based on the assumption that any data consists of different intrinsic modes of oscillations. Each intrinsic mode, linear or nonlinear, represents a simple oscillation. At any given time, the data may have many different coexisting modes of oscillation, one superimposing on the others. As a result, the waveform appears to be complicated. Each of these simple oscillatory modes is represented by an intrinsic mode function (IMF) with the following characteristics:

(1) in the whole dataset, the number of extrema and the number of zero-crossings must either be equal or differ at most by one, and

(2) at any point, the mean value of the envelope defined by the local maxima and the envelope defined by the local minima is zero.

IMF represents a simple oscillatory mode as a counterpart to the simple harmonic function, but it is much more general: instead of constant amplitude and frequency, as in a simple harmonic component, the IMF can have variable amplitude and frequency as a function of time. The total number of IMF components is close to $\log _{2} N$, where $N$ is the total number of data points. ${ }^{5)}$

The sifting process for a given data $x(t)$ is as follows: First, identify the local extrema (maxima and minima) and then obtain the upper and lower envelopes by connecting the local maxima and local minima using cubic spline method, respectively. Their mean is designated as $m_{1}$, and the difference between the data and $m_{1}$ is the first component $h_{1}$, which is a candidate for IMF,

$$
h_{1}=x(t)-m_{1}
$$

Ideally, $h_{1}$ should satisfy the definition of an IMF. The procedure should have made $h_{1}$ symmetric and all its maxima positive and all its minima negative. However, even if the sifting procedure is perfect, a gentle hump on a slope can be amplified to become a local extremum in changing the local zero from rectangular to a curvilinear coordinate system. The sifting process serves two purposes: (1) to eliminate the riding waves; and (2) to make the wave profiles more symmetric. While the first purpose must be achieved for the Hilbert Transform to give a meaningful instantaneous frequency, the second purpose must also be achieved in case the neighboring wave amplitudes have too large disparity. ${ }^{5)}$ The sifting process has to be repeated as many times as it is required to reduce the extracted signal to an IMF. In the subsequent sifting processes, $h_{1}$ is treated as the data for the next round of sifting. After repeating the sifting process $k$ number of times, $h_{1 k}$ is obtained

$$
\begin{gathered}
h_{11}=h_{1}-m_{11} \\
h_{12}=h_{11}-m_{12} \\
h_{13}=h_{12}-m_{13} \\
\vdots \quad \quad \vdots \\
h_{1 k}=h_{1(k-1)}-m_{1 k} .
\end{gathered}
$$

If $h_{1 k}$ satisfies the conditions for an IMF, then $h_{1 k}$ is designated as $c_{1}$

$$
c_{1}=h_{1 k},
$$

where $c_{1}$ is the first IMF component from the data. It should be noted that $c_{1}$ will contain the finest scale or the shortest period component of the signal. The next step is to remove $c_{1}$ from the rest of the data by

$$
r_{1}=x(t)-c_{1}
$$

where $r_{1}$ is called residue, which contains all longer period variations in the data. $r_{1}$ is treated as a new data set and subjected to the same sifting procedure described above. This procedure is repeated to all the subsequent $r_{j}$ 's and will result as

$$
\begin{gathered}
r_{2}=r_{1}-c_{2} \\
r_{3}=r_{2}-c_{3} \\
\vdots \\
r_{n}=r_{(n-1)}-c_{n} .
\end{gathered}
$$

The sifting should stop when the residue, $r_{n}$, becomes a monotonic function or a function containing only a single extrema, from which no more IMF can be extracted. Thus, the sifting process produces a decomposition of the data into $n$-intrinsic modes and a residue $r_{n}$

$$
x(t)=\sum_{j=1}^{n} c_{j}+r_{n} .
$$

In the book by Huang and Shen, ${ }^{5)}$ the EMD was proposed as an adaptive time-frequency data analysis method and proved that it was quite flexible in a wide range of applications for extracting signals from data generated in noisy non-linear and non-stationary processes. However, one of the major drawbacks of EMD is the frequent appearance of mode mixing, which is defined as a single Intrinsic Mode Function either consisting of widely incongruent scales or a signal of similar scale residing in different IMF components. To overcome the mode mixing problem, the Ensemble Empirical Mode Decomposition was proposed.

\section{(b) Ensemble Empirical Mode Decomposition (EEMD)}

Ensemble Empirical Mode Decomposition is a noiseassisted data analysis method which was proposed by $\mathrm{Wu}$ and Huang ${ }^{7)}$ as an improvement of the EMD. This method defines the true IMF components as the mean of an ensemble of trials where in each trial consists of the signal plus white noise of finite amplitude. Like EMD, this method separates the scale naturally without any a priori subjective criterion selection. EEMD is based on the recent studies of the statistical properties of white noise by. ${ }^{8,9)}$ These studies showed that the EMD is an adaptive dyadic filter bank when applied to white noise, that is, the Fourier spectra of various IMFs collapse to a single shape along the axis of logarithm of period or frequency. When the data is not pure noise, some scales could be missing and may cause mode mixing to occur.

The principle behind EEMD is described as follows: the finite amplitude white noise added to the signal covers the whole time-frequency space uniformly with the constituting components of different scales. Although adding white noise 
may result in smaller signal-to-noise ratio, the added white noise serves as a uniform reference scale where the bits of the signal are automatically projected onto the proper scales to facilitate EMD. Thus, the low signal-to-noise ratio does not affect the decomposition method but actually enhances it to avoid mode mixing. ${ }^{7)}$

\subsubsection{Hilbert spectral analysis (HSA)}

Nonlinear processes need special treatment. Aside from the periodicity, the detailed dynamics in the processes from the data need to be understood well because one of the typical characteristics of nonlinear process is their intra-wave frequency modulation, which indicates the instantaneous frequency (IF) change within one oscillation cycle. As Hung et $a{ }^{4)}$ pointed out, this intra-frequency variation is the hallmark of nonlinear systems. The easiest way to obtain the instantaneous frequency is by using the Hilbert transform, through which the complex conjugate $y(t)$ of any real valued function $x(t)$ of $L^{P}$ class can be determined by

$$
y(t)=H[x(t)]=\frac{1}{\pi} P V \int_{-\infty}^{+\infty} \frac{x(\tau)}{t-\tau} d \tau,
$$

where $P V$ indicates the principal value of the singular integral. With the use of Hilbert transform, the analytic signal is defined as

$$
z(t)=x(t)+i y(t)=a(t) e^{i \theta(t)}
$$

where

$$
a(t)=\sqrt{x^{2}+y^{2}}, \quad \text { and } \quad \theta(t)=\arctan \left(\frac{y}{x}\right) .
$$

The instantaneous amplitude is given by $a(t)$, the phase function by $\theta(t)$, and the instantaneous frequency is given by

$$
\omega(t)=\frac{\mathrm{d} \theta(t)}{\mathrm{d} t}
$$

Hilbert Spectral Analysis is a signal analysis method applying the Hilbert transform to compute the instantaneous frequency (IF) of signals according to eq. (10). After performing the Hilbert transform on each signal, in this case the IMFs, the data can be expressed in the following form

$$
z(t)=\sum_{j=1}^{n} a_{j}(t) \exp \left(i \int \omega_{j}(t) \mathrm{d} t\right),
$$

where $a_{j}(t)$ and $\omega_{j}(t)$ are the amplitude and frequency as a function of time for the $j^{\text {th }}$ IMF, respectively. From this expression the instantaneous frequency and amplitude as a function of time can be represented in a three-dimensional plot. This can also be represented in two-dimensional plot in which the amplitude is contoured on the frequency-time plane. The frequency-time distribution of the amplitude is designated as the Hilbert Amplitude Spectrum (HAS) or simply Hilbert Spectrum (HS).

\subsection{Image reconstruction}

The waveform data were evaluated through reconstruction calculations to determine the location, distribution and approximate size/dimension of acoustic reflectors and scatterers (possible defects or anomalies) in the concrete specimen being tested. In this study, the image reconstruction was done on both the processed and unprocessed waveform data for comparison. The image reconstruction was simulated in a 2D matrix where in each pixel is in Cartesian coordinate system. The position coordinates of the transmitting $\left(\mathrm{x}_{\mathrm{T}}, \mathrm{y}_{\mathrm{T}}\right)$ and the receiving $\left(\mathrm{x}_{\mathrm{R}}, \mathrm{y}_{\mathrm{R}}\right)$ transducers were in accordance with the above mentioned coordinate system. Figure 2 shows the schematic diagram for the simulation of wave propagation and reflection at a specific pixel $\left(\mathrm{x}_{\mathrm{i}}, \mathrm{y}_{\mathrm{i}}\right)$. At a particular transmitter location, only the pixels that are located within the radiation zone of the incident wave are considered in the numerical superposition. From the transmitting transducer, the incident wave propagates towards the pixel being considered and it is assumed that this pixel will reflect the wave towards the receiving transducers. The time of flight (TOF) is defined as the time to travel from the transmitting transducer to the pixel and from the pixel to the receiving transducer. TOF is given by the formula

$$
T O F=\frac{D_{\text {incident }}+D_{\text {reflected }}}{V},
$$

where

TOF: time of flight (s)

$D_{\text {incident }}$ : distance or length of the incidence path (m)

$D_{\text {reflected }}$ : distance or length of the reflected path (m)

$V$ : ultrasonic velocity through the concrete specimen $(\mathrm{m} / \mathrm{s})$. For all the waveforms, the SAFT algorithm numerically superimposes the amplitude value corresponding to the computed TOF to the specific pixel $\left(\mathrm{x}_{\mathrm{i}}, \mathrm{y}_{\mathrm{i}}\right)$ as given by eq. (13). This procedure is done for all the pixels within the radiation zone. The summation of the amplitude values gives an image wherein a pixel with high intensity indicates the location of a reflector.

$$
S\left(x_{i}, y_{i}\right)=\sum_{n=1}^{N} A_{n}(T O F)
$$

where

$S\left(x_{i}, y_{i}\right)$ : summation of amplitude values for pixel $i$

$A_{n}(T O F)$ : amplitude value of the $n^{\text {th }}$ waveform corresponding to the computed TOF

$N$ : total number of recorded waveform data

\section{Results and Discusion}

The waveforms obtained from the 8 receiver positions at a particular transmitter location were normalized with respect to the input waveform. Figure 3 shows the normalized waveforms at the location where the transmitter is directly above the embedded steel rod. It can be observed that each of these waveforms contains a lot of oscillations, making it very difficult to analyze and interpret. A SAFT image using all the 168 normalized waveforms is shown in Fig. 4. The reflection from the bottom surface of the concrete specimen is not clearly depicted from the reconstructed image. Moreover, the grouted steel rod cannot be located from this SAFT image.

After applying EEMD to the 168 normalized waveforms, each individual waveform was decomposed into their respective IMFs and residual function. One of the obtained waveforms and its IMF components are shown in Fig. 5. The label " $10-7$ " means that the transmitting transducer is located 


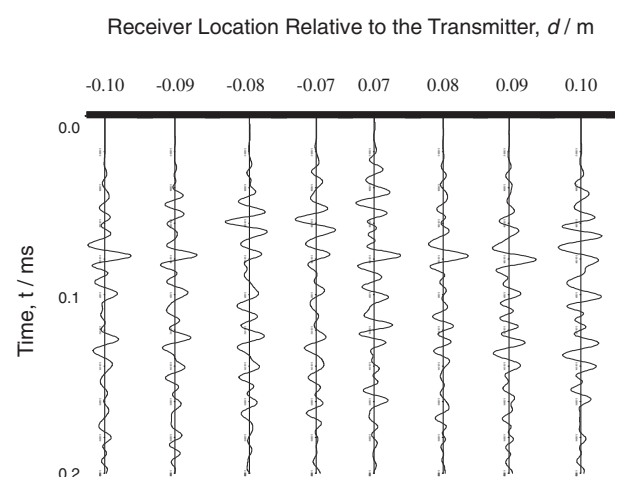

Fig. 3 Normalized waveforms.

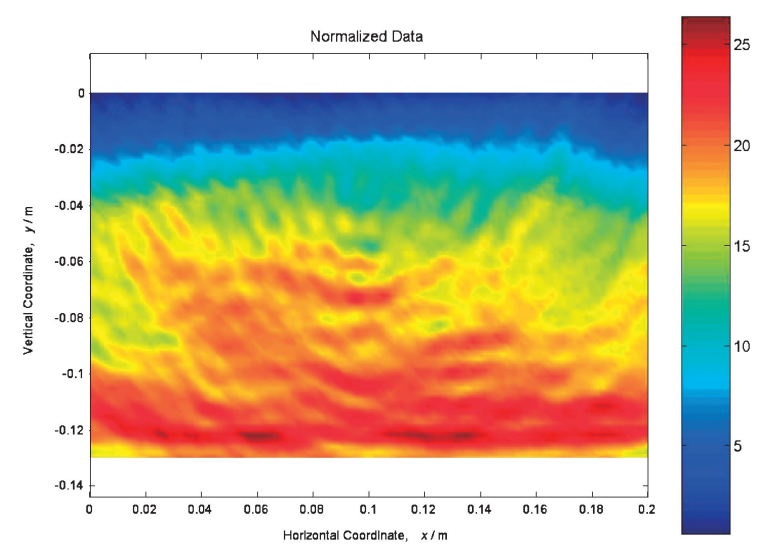

Fig. 4 SAFT image of normalized data.
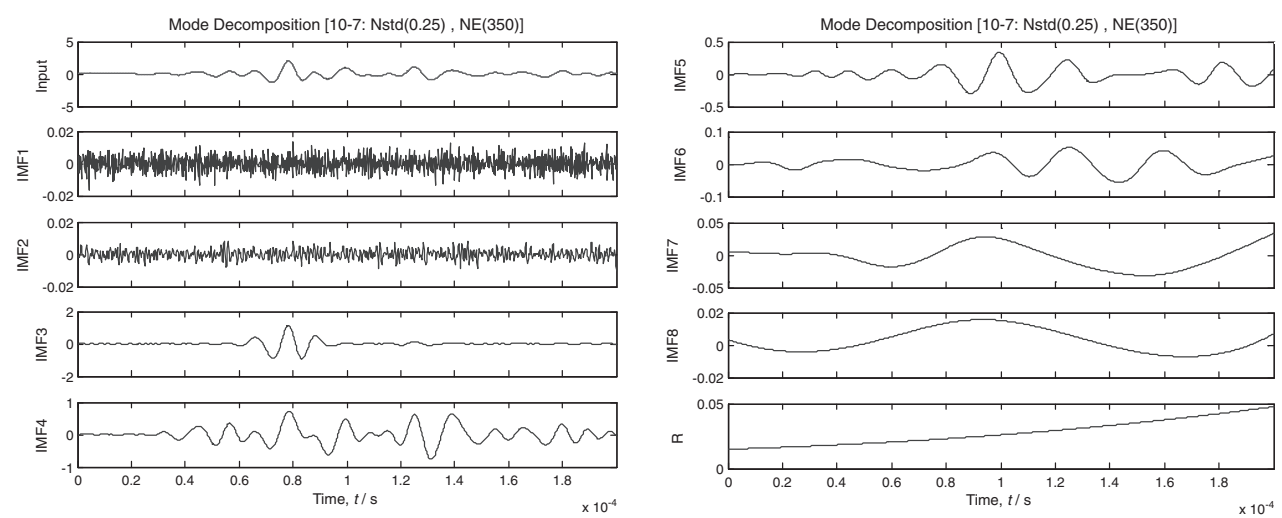

Fig. 5 Mode decomposition (EEMD) of one of the obtained waveforms.

(a)

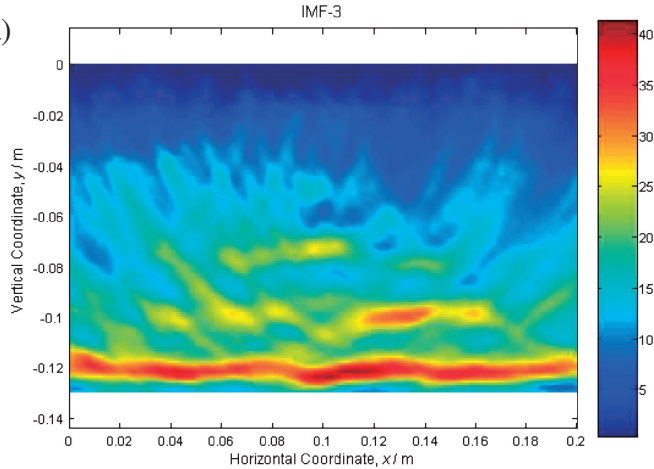

(b)

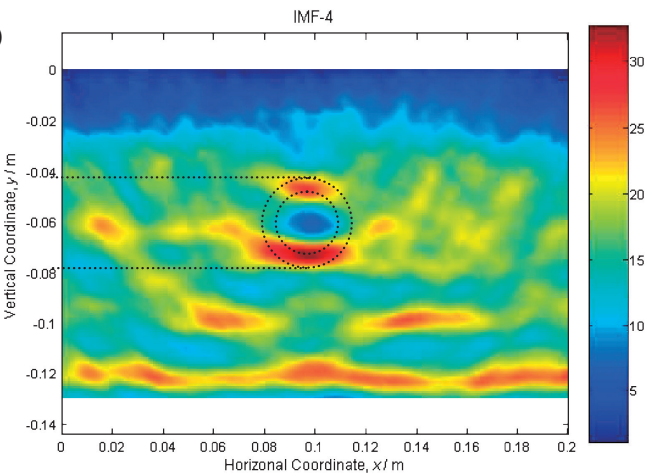

Fig. 6 SAFT image of the processed data. (a) Image reconstruction using IMF3, (b) Image reconstruction using IMF4.

at position "10" (directly above the grouted steel rod) and the receiving transducer is located at position "7" which is $100 \mathrm{~mm}$ to the left of the transmitting transducer. Among the eight IMFs, it can be observed that IMF3 and IMF4 are the most dominant in terms of amplitude. It should be noted that the first two IMFs, with high frequencies, correspond to the noise added to the signal during the EEMD procedure. For all the waveform data, the SAFT images for each IMF were obtained. Figure 6 shows the corresponding SAFT images for IMF3 and IMF4. In Fig. 6(a), the bottom surface of the concrete specimen is well defined by the high reflection intensity pixels located approximately $0.12 \mathrm{~m}$ from the top surface. On the other hand, as shown in Fig. 6(b), there are several regions with high reflection intensity but the image of the grouted steel rod is manifested by strong reflection intensity from its top and bottom surfaces and very low reflection intensity from its interior. It should be noted that the very low reflection intensity simply means that the interior of the steel rod is relatively homogeneous compared to the surrounding concrete. At this point, the corresponding images for the concrete bottom surface and the grouted steel rod were easy to indentify since their actual locations are known. However, for the other regions with high intensity reflection intensity (artifacts) can be verified once the concrete slab is cut to obtain its cross-section.

The IMF components were then subjected to Hilbert Spectral Analysis in order to obtain the frequency and amplitude variation as a function of time. Figure 7 shows 
(a)

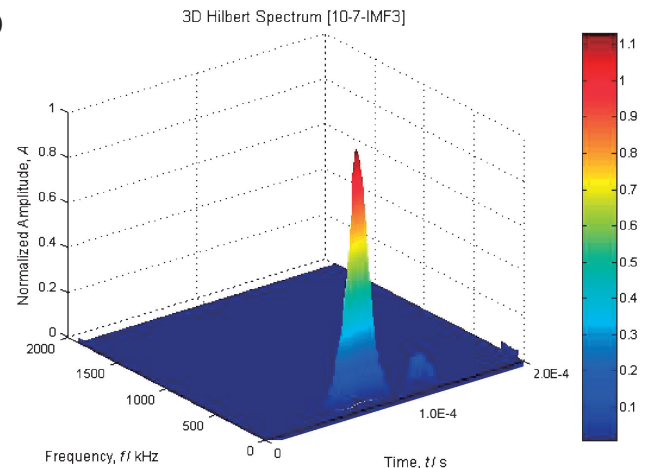

(b)

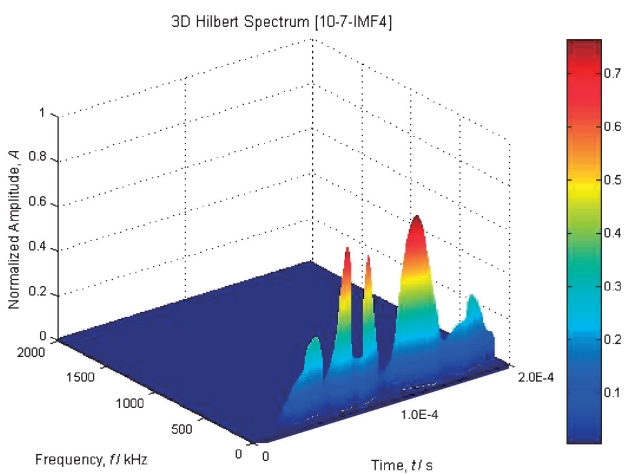

Fig. 7 Frequency and amplitude variation as a function of time. (a) Hilbert spectrum for IMF3, (b) Hilbert spectrum for IMF4.

the 3D Hilbert Spectrum for IMF3 and IMF4 of Fig. 5. With these figures, the time of flight and the frequency corresponding to the reflected signals can be determined and/or verified. In Fig. 7(a), the reflection from the concrete buttom surface is represented by a peak at frequency $\approx$ $100 \mathrm{kHz}$ and at time $\approx 78 \mu \mathrm{s}$. In Fig. 7(b), the reflection from the buttom surface of the grouted steel rod is represented by the first peak at frequency $\approx 80$ to $90 \mathrm{kHz}$ and at time $\approx 47 \mu$ s. Aparently, the top surface of the grouted steel rod is not seen in this figure. However, it should be noted that these 3D Hilbert spectra are for IMF 3 and IMF 4 of the waveform "10-7" only.

\section{Conclusion}

The Hilbert-Huang Transform based on Ensemble Empirical Mode Decomposition was used as a signal processing tool for interpreting and analyzing the ultrasonic waveforms obtained from a concrete specimen with an embedded steel rod. Reflections from the bottom surface as well as the steel rod were successfully depicted in the image reconstruction using the Synthetic Aperture Focusing Technique. Therefore, anomaly or defect detection in concrete structures through ultrasonic imaging was greatly improved by the combination of HHT and SAFT. Moreover, an efficient and accurate evaluation of the concrete structure interior was made possible with these combined techniques.

\section{Acknowledgement}

The authors would like to thank Dr. Norden E. Huang, Dr. Zhaohua Wu and Mr. Seng-Chung Su for the help in understanding the implementation of HHT. Also, the principal author would like to thank the Japan Society for the Promotion of Science (JSPS) and the Japanese Government (MEXT) for providing scholarship for his graduate studies at Tokyo Institute of Technology.

\section{REFERENCES}

1) O. Buyukozturk: NDT \& E Int. 31 (1998) 233-243.

2) Repair-Evaluation-Maintenance-Rehabilitation Research Program: REMR Technical Note CS-ES 1.10 (1991).

3) A. Abbate, C. M. DeCusatis and P. K. Das: Wavelets and Subbands Fundamentals and Applications, (Birkhauser, Boston, 2002) pp. 18-20.

4) N. E. Huang, Z. Shen, S. R. Long, M. C. Wu, H. H. Shih, Q. Zheng, N. C. Yen, C. C. Tung and H. H. Liu: Proc. R. Soc. Lond. Ser. A 454 (1998) 903-995.

5) N. E. Huang and S. S. P. Shen: Hilbert-Huang Transform and Its Applications, (World Scientific Publishing Co. Pte. Ltd., Singapore, 2005) pp. 1-15.

6) M. Schickert, M. Krause and W. Muller: J. Mater. Civil Eng. 15 (2003) 235-246.

7) Z. Wu and N. E. Huang: Adv. Adaptive Data Analy. 1 (2009) 1-41.

8) P. Flandrin, G. Rilling and P. Goncalves: Signal Process. Lett. 11 (2004) 112-114.

9) Z. Wu and N. E. Huang: Proc. R. Soc. Lond. Ser. A 460 (2004) 15971611 . 